Photosynthesis Research 40: 55-66, 1994.

(C) 1994 Kluwer Academic Publishers. Printed in the Netherlands.

Regular paper

\title{
Spectroscopic characterization of reaction centers of the (M)Y210W mutant of the photosynthetic bacterium Rhodobacter sphaeroides
}

\author{
Susana Shochat ${ }^{1}$, Thomas Arlt ${ }^{2}$, Christof Francke ${ }^{1}$, Peter Gast ${ }^{1}$, Paula I. van Noort ${ }^{1}$, Stephan C. \\ M. Otte ${ }^{1}$, Hans P. M. Schelvis ${ }^{1}$, Stefan Schmidt ${ }^{2}$, Erik Vijgenboom ${ }^{3}$, Jacobien Vrieze ${ }^{1}$, Wolfgang \\ Zinth $^{4}$ and Arnold J. Hoff ${ }^{1}$ \\ ${ }^{1}$ Department of Biophysics, Huygens Laboratory, Leiden University, P.O. Box 9504, 2300 RA \\ Leiden, The Netherlands; ${ }^{2}$ Technische Universität München, Physik Department E 11, James Franck \\ Str., 85748 Garching, Germany; ${ }^{3}$ Department of Chemistry, Gorlaeus Laboratory, Leiden University, \\ P.O. Box 9502, 2300 RA Leiden, The Netherlands; ${ }^{4}$ Ludwig-Maximilians-Universität München, \\ Institut für Medizinische Optik, Barbarastr. 16, 80797 München, Germany
}

Received 15 July 1993; accepted in revised form 7 December 1993

Key words: absorption spectrum, ADMR, charge separation, fluorescence spectrum, reaction center, mutant

\begin{abstract}
The tyrosine-(M)210 of the reaction center of Rhodobacter sphaeroides 2.4 .1 has been changed to a tryptophan using site-directed mutagenesis. The reaction center of this mutant has been characterized by low-temperature absorption and fluorescence spectroscopy, time-resolved sub-picosecond spectroscopy, and magnetic resonance spectroscopy. The charge separation process showed bi-exponential kinetics at room temperature, with a main time constant of $36 \mathrm{ps}$ and an additional fast time constant of 5.1 ps. Temperature dependent fluorescence measurements predict that the lifetime of $\mathrm{P}^{*}$ becomes $4-5$ times slower at cryogenic temperatures. From EPR and absorbance-detected magnetic resonance (ADMR, LD-ADMR) we conclude that the dimeric structure of $\mathbf{P}$ is not significantly changed upon mutation. In contrast, the interaction of the accessory bacteriochlorophyll $\mathrm{B}_{\mathrm{A}}$ with its environment appears to be altered, possibly because of a change in its position.
\end{abstract}

Abbreviations: ADMR - absorbance-detected magnetic resonance; LDAO - N, N dimethyl dodecyl amine-N-oxide; RC - reaction center; LD-ADMR - linear-dichroic absorbance-detected magnetic resonance; P - primary donor; B - accessory bacteriochlorophyll; $\Phi$ - bacteriopheophytin

\section{Introduction}

The primary reactions in bacterial photosynthesis generate chemical energy by a charge separation across a membrane-bound pigment-protein complex, the reaction center (RC). The X-ray structure of the $\mathrm{RC}$ of Rhodopseudomonas viridis (Michel and Deisenhofer 1986) and Rhodobacter (Rb.) sphaeroides $R$-26 (Allen et al. 1987b) has been solved. It shows that the electron transfer cofactors are arranged in two branches, $\mathrm{A}$ and $\mathrm{B}$, related by a pseudo- $\mathrm{C} 2$ symmetry. The axis of symmetry runs from a dimer of bacteriochlorophyll (BChl) molecules (the primary electron donor, $\mathrm{P}$ ), at the periplasmic side of the membrane, to an iron atom at the cytoplasmic side. Each branch contains, in addition to one $\mathrm{BChl}$ of $\mathrm{P}$, a $\mathrm{BChl}$ molecule, located near $\mathrm{P}\left(\mathrm{B}_{\mathrm{A}}\right.$ and $\left.\mathrm{B}_{\mathrm{B}}\right)$, a bacteriopheophytin molecule $\left(\Phi_{A}\right.$ and $\left.\Phi_{B}\right)$ and a quinone $\left(Q_{A}\right.$ and $\left.Q_{B}\right)$. Spectroscopic studies 
have revealed that electron transfer proceeds only through chain A (reviewed in Kirmaier and Holten 1987).

The cofactors are embedded in a polypeptide complex comprising three subunits (L, M and $\mathrm{H})$. The cofactor chromophores of the active branch (A), and $Q_{B}$ are associated with the $L$ subunit, while those of the inactive branch (B) and $Q_{A}$ are associated with the $M$ subunit; the $H$ subunit caps the $\mathrm{L}$ and $\mathrm{M}$ unit at the quinone sites.

Several amino acids in the vicinity of the cofactors break the symmetry of the complex. It has been suggested that these amino acids play a role in both the unidirectionality (only one active branch) and the highly efficient electron transfer. They may, for example, affect the energy levels of the electron transfer components or the coupling between the different intermediates.

One of the most striking symmetry-breaking residues is tyrosine (M) $\mathrm{Y} 210$, which, although part of the $M$ subunit, is located very close to $P$, $\mathrm{B}_{\mathrm{A}}$, and $\Phi_{\mathrm{A}}$. This residue is conserved in $R b$. capsulatus, $R b$. sphaeroides, Rhodospirillum rubrum and Rhodopseudomonas viridis (Williams et al. 1986). The symmetry-related amino acid in the $\mathbf{L}$ polypeptide is phenylalanine at position L181. In Chloroflexus aurantiacus, the tyrosine is replaced by leucine (Shiozawa et al. 1989). Charge separation in this bacterium is more than two times slower than in the other bacteria mentioned (Becker et al. 1991, Hamm et al. 1993). Calculations of the electrostatic energy of the reactants involved in charge separation, suggest that the interaction of $\mathrm{P}$ and $\mathrm{B}_{\mathrm{A}}$ with the polar tyrosine, (M)Y210, lowers the energy of the state $\mathrm{P}^{+} \mathrm{B}_{\mathrm{A}}^{-}$, rendering electron transfer through the A-branch more favorable than that along the B-branch (Parson et al. 1990).

To understand the role of (M)Y210, site-directed mutagenesis techniques have been used to change this residue to phenylalanine (Gray et al. 1990, Nagarajan et al. 1990, Finkele et al. 1990), leucine (Gray et al. 1990, Finkele et al. 1990) and isoleucine (Nagarajan et al. 1990) in $R b$. sphaeroides, and to phenylalanine, histidine and threonine in $R b$. capsulatus (Chan et al. 1991). Except for the histidine (which is aromatic and polar like the tyrosine), it was found that in all cases the rate of charge separation was lower in the mutants than in the wild type, indicating that (M)Y210 plays an important role in the initial electron transfer. Extensive research has been carried out to characterize the mutants mentioned. Considering the charge-separation rate in different mutants of $R b$. capsulatus, Chan et al. (1991) concluded that both the hydroxyl group and the aromatic ring of the tyrosine are important for efficient electron transfer through the A-branch. Using Raman spectroscopy, Mattioli et al. (1991) concluded that the tyrosine is not hydrogen bonded to $\mathrm{P}$, contrary to a suggestion based on the X-ray structure (Allen et al. 1987a). Thus, a direct interaction with $P$ seems to be excluded. Possibly, the phenolic residue acts on electron transfer by influencing the polarity of the electrostatic environment of P. Nagarajan et al. (1990) performed studies on the temperature dependence of the rate of charge separation in two mutants of $R b$. sphaeroides. Their results suggest that the mutations indeed raise the energy level of $\mathrm{P}^{+} \mathbf{B}_{\mathrm{A}}^{-}$, making the initial electron transfer reaction thermally activated at room temperature.

In the course of CP/MAS NMR experiments on ${ }^{13} \mathrm{C}$-tyrosine labelled RCs of $R b$. sphaeroides R-26 (Fischer et al. 1992), it was decided to replace (M)Y210 with another amino acid for the purpose of peak assignment. Tryptophan was chosen because in principle it allows doublelabelling $\left({ }^{13} \mathrm{C},{ }^{15} \mathrm{~N}\right)$ experiments. In this communication we report on the physico-chemical properties of the (M)Y210W mutant, characterizing its RC by low-temperature absorption and fluorescence spectroscopy, time-resolved absorbance-difference spectroscopy and magnetic resonance spectroscopy, comparing the results with those reported for other (M)Y210 mutants. Part of this work has appeared in preliminary form (Shochat et al. 1992).

\section{Materials and methods}

The restriction endonucleases BamHI, SalI and XhoI were obtained from Amersham Corporation. Kanamycin and tetracycline were from Sigma. The deletion mutant of $R b$. sphaeroides 2.4.1( $\Delta \mathrm{LM} 1)$, the expression vector, which is a broad host range plasmid pRK404 containing the 
puf operon (pRKNEB1), and Escherichia (E.) coli S17-1 were made available by Prof. G. Feher (Paddock et al. 1989). Q-sepharose and DEAE sephacel were obtained from Pharmacia; $\mathrm{N}, \mathrm{N}$ dimethyl dodecyl amine-N-oxide (LDAO) from Fluka Chemie.

The $R b$. sphaeroides deletion strain complemented with the plasmid bearing wild type or mutated genes was grown semi-aerobically in the dark, on rich medium, in the presence of kanamycin $(25 \mathrm{mg} / \mathrm{ml})$ and tetracycline $(2.5 \mathrm{mg} / \mathrm{ml})$ (Paddock et al. 1989).

The mutation was incorporated into the gene using a synthetic oligonucleotide carrying the changed codon TAC(tyr) $\rightarrow$ ACC(trp). The 1 $\mathrm{Kb}$ SalI-BamHI fragment, containing the puf $\mathrm{M}$ gene, was cloned into the bacteriophage M13 mp19. The SalI-BamHI fragment was isolated from the pRKENB1, which is a derivative of the pRKGln (Paddock et al. 1989) with a BamHI site created downstream from puf M. The M13 clone was used as a template for site-directed mutagenesis according to Kunkel (1987). Sequence analysis confirmed that only this mutation was present. The mutated gene was recloned in the expression vector pRKENB1 as a XhoI-BamHI fragment and was transformed into $E$. coli S17-1. This $E$. coli strain carries the genes necessary for plasmid transfer via conjugation. It was then mated with the deletion mutant of $R b$. sphaeroides as described by Paddock et al. (1989). Several exconjugants were picked and checked for the plasmid. To confirm that the desired mutation in the puf $\mathrm{M}$ gene was introduced into $R b$. sphaeroides, the pRKNEB1 plasmid bearing the mutation was isolated from $R b$. sphaeroides and the BamHI-SalI fragment was sequenced after being cloned again in M13 bacteriophage.

Chromatophores were obtained from a sonicated cell suspension. Sonication was performed with a $300 \mathrm{~W}$ sonifier for $10 \mathrm{~min}$ at $4{ }^{\circ} \mathrm{C}$. Isolated chromatophores were resuspended in $100 \mathrm{mM}$ phosphate buffer, $\mathrm{pH} 7.5$. The RCs were extracted with $0.25 \%$ LDAO by two consecutive incubations ( $45 \mathrm{~min}$ and $30 \mathrm{~min}$ ) of chromatophores resuspended to a concentration of $\mathrm{OD}_{865}=30 / \mathrm{cm}$. After the second incubation, the RCs were precipitated with $26 \%$ ammonium sulfate, resuspended in $10 \mathrm{mM}$ Tris- $\mathrm{HCl} \mathrm{pH} 8$ containing $0.1 \%$ LDAO and desalted using a sephadex G-50 column. The solution was then loaded at room temperature on a Q-sepharose column which was washed with $10 \mathrm{mM}$ Tris- $\mathrm{HCl}$ pH 8 containing $0.1 \%$ LDAO (TL buffer), after which the RCs were eluted using a $100-600 \mathrm{mM}$ $\mathrm{NaCl}$ gradient. This purification procedure was necessary in order to separate the RCs from a minor contamination with light-harvesting complex that sometimes was extracted together with the RCs. After dialysis against TL buffer, the RCs were passed through a DEAE sephacel column at $4{ }^{\circ} \mathrm{C}$, washed, and eluted with a $100-$ $300 \mathrm{mM} \mathrm{NaCl}$ gradient. The RCs were then dialyzed against TL buffer and concentrated over a $100 \mathrm{kD}$ Amicon filter. The $\mathrm{A}_{280} / \mathrm{A}_{802}$ and $\mathrm{A}_{280} /$ $\mathrm{A}_{806}$ ratios for the wild type and the mutant, were between 1.2 and 1.4 , respectively. Throughout this work 'wild type' refers to the deletion mutant complemented with wild-type genes.

Absorption, fluorescence emission and fluorescence excitation spectroscopy were performed using a single-beam spectrophotometer, equipped with a personal computer for data analysis as described by Otte et al. (1991). The spectral resolution was $0.5 \mathrm{~nm}$ and $3.0 \mathrm{~nm}$ for absorption and fluorescence measurements, respectively. The intensity of the excitation light was less than $50 \mu \mathrm{W} / \mathrm{cm}^{2}$. Glycerol $(60 \% \mathrm{v} / \mathrm{v})$ was added to all samples used in low-temperature experiments. The final absorbance was always less than $0.2 \mathrm{OD}$ at the peak of the accessory BChl absorption band ( $802 \mathrm{~nm}$ for the wild type and $806 \mathrm{~nm}$ for the mutant).

The transient absorption data were obtained with a sub-picosecond laser system operating at $50 \mathrm{~Hz}$ (Dressler et al. 1991, Schmidt et al. 1993). The sample was held in a $1 \mathrm{~mm}$ cuvette with an optical density of about 0.5 at $870 \mathrm{~nm}$. Excitation was at $870 \mathrm{~nm}$; about $10 \%$ of the RCs were excited per laser pulse. Stirring of the sample prevented accumulation of oxidized $P$ in the excitation beam. The width of the instrumental response function was around $300 \mathrm{fs}$. For measurements on a slower time scale, a ps spectrophotometer was used with a pulse width of 30 ps as described (Schelvis et al. 1992); optical changes on a ms time scale were measured with an absorption-difference spectrophotometer de- 
scribed by Visser (1975). All optical experiments were performed at room temperature. EPR measurements were carried out at about $120 \mathrm{~K}$ with an X-band Varian E-9 spectrometer as described by Gast and Hoff (1979).

The ADMR (Absorbance-Detected Magnetic Resonance) and LD-ADMR (Linear-Dichroic ADMR) set-ups are described in detail by Den Blanken et al. (1984). A single tungsten-iodine lamp is used for continuous excitation and as the source of the detection beam. Amplitude modulation of the microwaves and subsequent phasesensitive detection of the resulting modulation in transmittance results in a Triplet-minus-Singlet $(\mathrm{T}-\mathrm{S})$ absorbance spectrum. The optical density of RCs used in these experiments was about 0.3 at $802 \mathrm{~nm}$. For reducing the primary electron acceptor $Q_{A}, 10 \mathrm{mM}$ sodium ascorbate was added, and the sample was frozen under illumination with white light. Glycerol $(66 \% \mathrm{~V} / \mathrm{V})$ was added to obtain a clear sample. The ADMR and (LD)-ADMR measurements were carried out at $1.2 \mathrm{~K}$.

\section{Results}

\section{Growth characteristics}

The mutant (M)Y210W was capable of photoheterotrophic growth tested at a light intensity of $50 \mathrm{~W} / \mathrm{m}^{2}$. This was determined by comparing the number of colonies on plates at several dilutions (starting from the same inoculum of cells grown semi-aerobically) under photoheterotrophic and chemoheterotrophic conditions. We found the same number of colonies under both conditions.

The RCs are photochemically active as demonstrated by the reversible bleaching of the longwavelength absorption band of $\mathbf{P}$ by actinic light (data not shown). The amplitude of the photobleaching at $865 \mathrm{~nm}$ in a suspension of chromatophores of mutant or wild type, containing equal concentration of bacteriochlorophyll, was the same for both. This indicates that the amount of RCs in the photosynthetic membranes of the (M)Y210W mutant is comparable to that of the wild type.

By monitoring the kinetics of the reversible photobleaching at $865 \mathrm{~nm}$ on a ms-time scale (data not shown), it was found that for mutant RCs at least $90 \%$ of the $Q_{A}$ sites and $60 \%$ of the $\mathrm{Q}_{\mathrm{B}}$ sites were occupied after the isolation procedure. In the wild type $R C$ virtually all $Q_{A}$ and $\mathrm{Q}_{\mathrm{B}}$ were still present.

\section{Low-temperature steady-state spectroscopy}

The absorption spectra of isolated RCs of the wild type and the (M)Y210W mutant are shown in Figs. 1A and 1B, respectively. Whereas some differences are detected at room temperature (dashed lines), they become more apparent at $6 \mathrm{~K}$ (solid lines). At this low temperature the $Q_{y}$ band of the accessory $\mathrm{BChl}$ is shifted to $806 \mathrm{~nm}$ for the mutant, compared to $802 \mathrm{~nm}$ for the wild type. The bacteriopheophytin $Q_{y}$ band showed a blue shift of $4 \mathrm{~nm}$ to $757 \mathrm{~nm}$. The exact position of the $Q_{y}$ band of the primary electron donor $P$ depended on sample preparation and varied from $896 \mathrm{~nm}$ to $900 \mathrm{~nm}$. This band was always found at $896 \mathrm{~nm}$ in RCs isolated from the wild
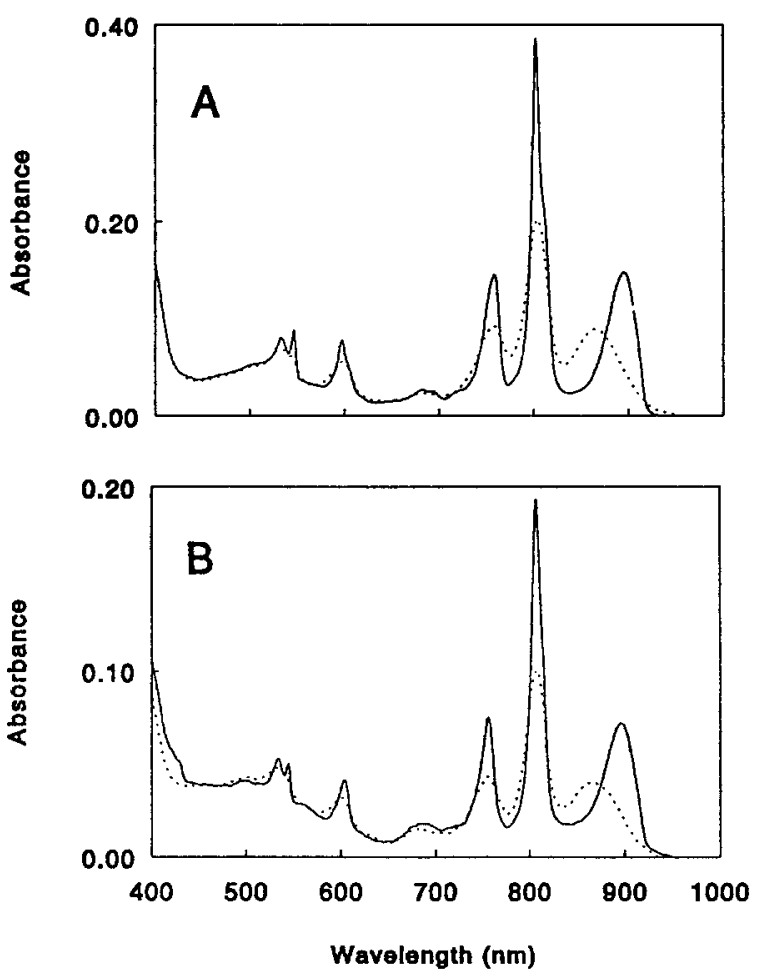

Fig. 1. Absorption spectra of isolated RCs of wild type (A) and the (M)Y210W mutant (B) at room temperature (dashed lines) and $6 \mathrm{~K}$ (solid lines). See 'Materials and methods' for the experimental conditions. 
type. In the $\mathrm{Q}_{\mathrm{x}}$ region the wild type has a band at $597 \mathrm{~nm}$, with a shoulder at $606 \mathrm{~nm}$, as revealed by the second derivative spectrum (not shown). The $597 \mathrm{~nm}$ band was attributed to the $Q_{x}$ transition of the $800 \mathrm{~nm} \mathrm{BChl}$. The $606 \mathrm{~nm}$ band was suggested to represent at least partly the $Q_{x}$ band of $P$, with possibly a contribution of the $Q_{x}$ band of $B_{B}$ (Kirmaier et al. 1985). Similar to the mutants discussed by Gray et al. (1990) and Mattioli et al. (1990), the $597 \mathrm{~nm}$ band of the wild type exhibits a red shift to $604 \mathrm{~nm}$ in the mutant; consequently it then overlaps with the component at $606 \mathrm{~nm}$. The absorption band of $\Phi_{\mathrm{A}}$ for the wild type was located at $547 \mathrm{~nm}$; for the mutant this band was blue shifted $2 \mathrm{~nm}$ to $545 \mathrm{~nm}$. The absorption band of $\Phi_{\mathrm{B}}$ at $535 \mathrm{~nm}$ is virtually unaffected by the tryptophan substitution.

The fluorescence emission spectra at $6 \mathrm{~K}$ of open RCs of the mutant and the wild type are given in Fig. 2 for excitation at the $B C h l Q_{x}$ band. The mutant showed a pronounced emis-
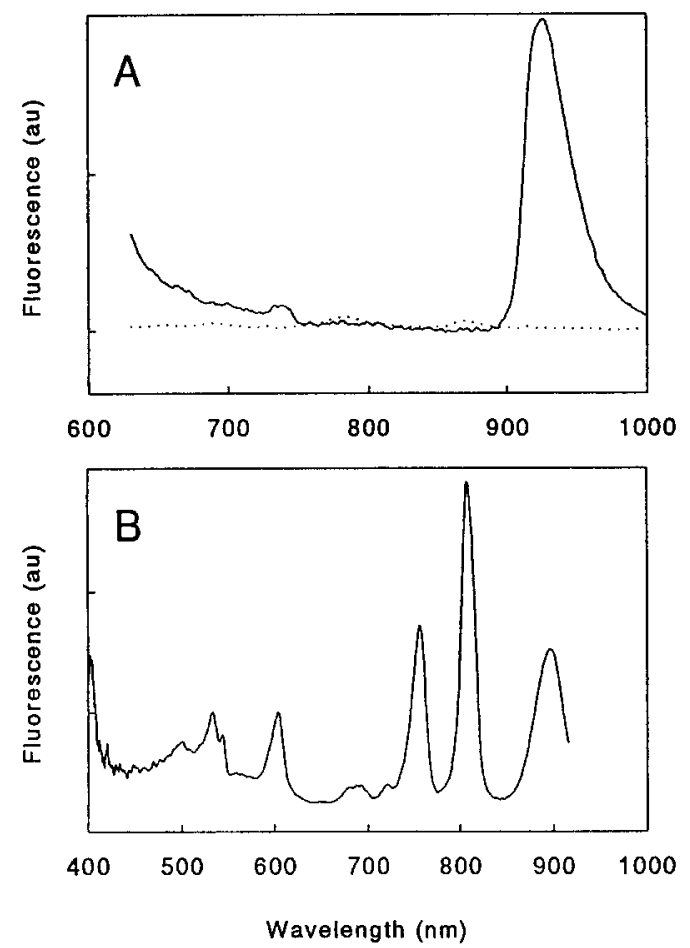

Fig. 2. (A) Fluorescence emission spectra of RCs of the wild type (dashed line) and the (M)Y210W mutant (solid line) at 6 $\mathrm{K}$ with excitation given at 597 and $603 \mathrm{~nm}$, respectively. (B) Fluorescence excitation spectrum of mutant RCs at $6 \mathrm{~K}$; the detection wavelength was $930 \mathrm{~nm}$. sion band at $926 \mathrm{~nm}$ (Fig. 2A), which we attribute to fluorescence from the excited singlet state of $\mathrm{P}, \mathrm{P}^{*}$. No fluorescence band at that wavelength was observed for the wild-type RCs, both at $6 \mathrm{~K}$ (Fig. 2A) and at room temperature (data not shown). The yield of the fluorescence was estimated using a suspension of chromatophores of Rhodospirillum rubrum as a standard, assuming a 1-5\% fluorescence yield for the chromatophores (Wang and Clayton 1971). The yield of the $926 \mathrm{~nm}$ emission band for the mutant was $0.1-0.6 \%$ at $6 \mathrm{~K}$, and four times lower at $298 \mathrm{~K}$. The yield at $6 \mathrm{~K}$ for the wild type was at least 20 times lower than that for the mutant.

The fluorescence excitation spectrum of the mutant at $6 \mathrm{~K}$ (Fig. 2B) is very similar to the low-temperature absorption spectrum, indicating that all RC pigments transfer the excitation energy efficiently to $P$ (Breton et al. 1986).

The EPR linewidth $\left(\Delta \mathrm{H}_{\mathrm{pp}}\right)$ of $\mathrm{P}^{+}$was $9.7 \pm$ $0.2 \mathrm{G}$ for both mutant and wild-type RCs.

\section{Primary charge separation}

The dynamics of the primary charge separation was studied by probing the lifetime of the excited electronic state $\mathbf{P}^{*}$ at room temperature at $920 \mathrm{~nm}$. Figure 3 shows the transient absorption curves of the wild type (Fig. 3A) and the (M)Y210W mutant (Fig. 3B) RC. The absorbance decrease at $920 \mathrm{~nm}$ at early delay times is due to stimulated emission from the excited electronic level of $\mathrm{P}^{*}$. Its decay rate reflects the depopulation of the excited primary donor. It is, therefore, to first approximation identical to the rate of charge separation.

The decay of $\mathbf{P}^{*}$ in the wild type was fitted to an exponential function with a time constant of $2.8 \mathrm{ps}$. The systematic deviation between fit and data points in the 10 ps regime may be reduced using two time constants (e.g. 2.3 ps and 7 ps as found in Hamm et al. (1993)). The late relative absorbance decrease can be fitted with a $220 \mathrm{ps}$ time constant. This time constant is due to the electron transfer from $\Phi_{\mathrm{A}}$ to $\mathrm{Q}_{\mathrm{A}}$ (Holzapfel et al. 1990). The decay of $P^{*}$ in the (M)Y210W mutant was best fitted by a sum of two exponentials with time constants of $5.1 \pm 0.8 \mathrm{ps}(20 \%)$ and $36.0 \pm$ $1.8 \mathrm{ps}(70 \%)$. The residual amplitudes $(6 \%$ in the wild type and $10 \%$ in the mutant), are due to 


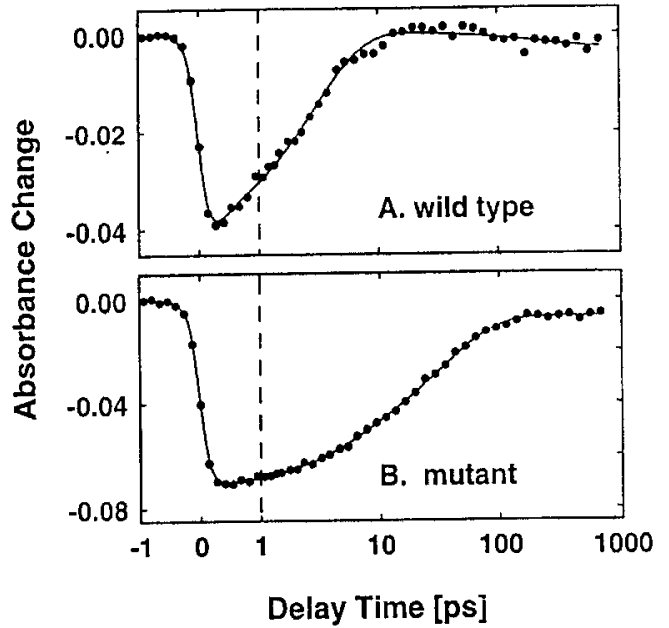

Fig. 3. Transient absorption kinetics of RCs isolated from the wild type (A) and the (M)Y210W mutant (B), measured at a probing wavelength of $920 \mathrm{~nm}$. The absorbance change in the wild type was fitted with a sum of two exponentials having a time constant of 2.8 ps and 220 ps. The kinetics in the (M)Y210W mutant was best fitted with two exponentials with a time constant of $5.1 \pm 0.8 \mathrm{ps}$ and $36.0 \pm 1.8 \mathrm{ps}$. The residual amplitudes at late delay times is due to $\mathrm{P}^{+} \mathrm{Q}_{\mathrm{A}}^{-}$. At early delay times $(t<1 \mathrm{ps})$, a linear timescale and, for later times $(t>1 \mathrm{ps})$, a logarithmic scale is used.

$\mathrm{P}^{+} \mathrm{Q}_{\mathrm{A}}^{-}$absorption (Van Noort et al., manuscript in preparation).

\section{$A D M R$ and $L D-A D M R$}

ADMR spectroscopy of the mutant $\mathrm{RC}$ at a detection wavelength of $905 \mathrm{~nm}$ (the peak of the long-wavelength absorption band at $1.2 \mathrm{~K}$ ) yielded two zero-field (zf) transitions of the primary donor triplet, $\mathrm{P}^{\mathrm{T}}$ (data not shown). The maximum of the $|\mathrm{D}|-|\mathrm{E}|$ transition was at $463 \mathrm{MHz}$; that of the $|D|+|E|$ transition at $656 \mathrm{MHz}$, where $\mathrm{D}$ and $\mathrm{E}$ represent the zero-field splitting (ZFS) parameters. For different detection wavelengths within the long-wavelength band (centered at $905 \mathrm{~nm}$ ), the zero-field frequencies shift with the detection wavelength, similar to the behavior found for RCs of $R b$. sphaeriodes R26 (Den Blanken et al. 1983) and RCs of other purple bacteria (J. Vrieze, unpublished results). This shift has been attributed to heterogeneity with respect to the exact configuration of the primary donor (Den Blanken et al. 1983). At the absorption peak of the T-S spectrum, which is attributed to accessory bacteriochlorophyll
(Scherer and Fischer 1987) and located at $808 \mathrm{~nm}$ for the wild type and $809 \mathrm{~nm}$ for the mutant, the shift of the zero-field frequencies with detection wavelength is very small. The ZFS parameters determined at this wavelength are $|\mathrm{D}|=189.5 \pm 0.310^{-4} \mathrm{~cm}^{-1}$ and $|\mathrm{E}|=32.2 \pm$ $0.210^{-4} \mathrm{~cm}^{-1}$ (Fig. 4). These values are virtually the same as found for wild-type $\mathrm{RCs}$ at the corresponding wavelength of $808 \mathrm{~nm}(|\mathrm{D}|=187.3$ $10^{-4} \mathrm{~cm}^{-1}$ and $|\mathrm{E}|=31.710^{-4} \mathrm{~cm}^{-1}$. The decay rates of the three triplet sublevels are also practically identical to those measured for the wild-type RCs (data not shown). Thus, the properties of the triplet state of the primary donor do not change on introducing the (M)Y210W mutation.

$\mathrm{T}-\mathrm{S}$ and $\mathrm{LD}(\mathrm{T}-\mathrm{S})$ spectra of wild-type and mutant RCs are shown in Fig. 5. The $T-S$ and $\mathrm{LD}(\mathrm{T}-\mathrm{S})$ spectra were measured at 463 and $656 \mathrm{MHz}$. Some differences are observed between the $\mathrm{T}-\mathrm{S}$ spectra of the mutant and the wild type. The positive $809 \mathrm{~nm}$ band of the mutant is lower in intensity than the corresponding band of the wild type and redshifted by $1 \mathrm{~nm}$, most probably because of a shift in the $\mathrm{S}_{1} \leftarrow \mathrm{S}_{0}$ transition in the absorption spectrum from 802 to $806 \mathrm{~nm}$, which leads to a stronger overlap of the $808 \mathrm{~nm}$ band with the adjacent negative band in the $\mathrm{T}-\mathrm{S}$ spectrum. The intensity of the $820 \mathrm{~nm}$ band is equal for the wild type and the mutant, which is in agreement with the identical peak wavelength and intensity of the $814 \mathrm{~nm}$ band in the deconvoluted $S_{1} \leftarrow S_{0}$ absorption spectrum of the two species. The low-energy band of the primary donor of the

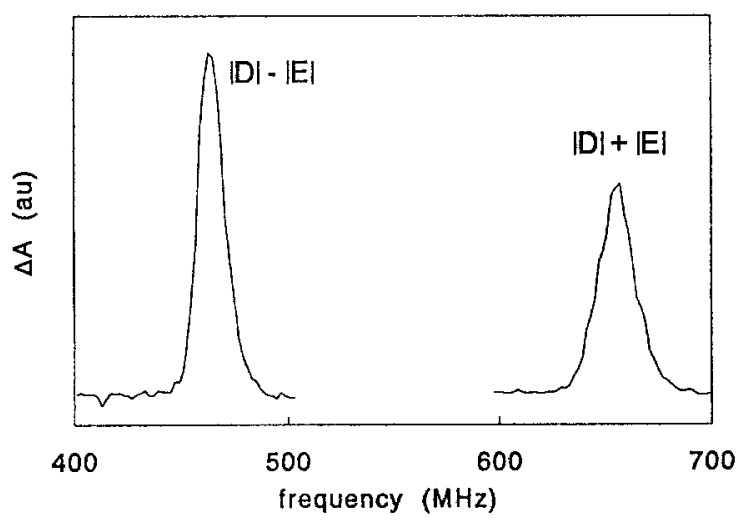

Fig. 4. ADMR-detected zero-field transitions of the mutant $\mathrm{RC}$ at $1.2 \mathrm{~K}$. The detection wavelength was $809 \mathrm{~nm}$. 


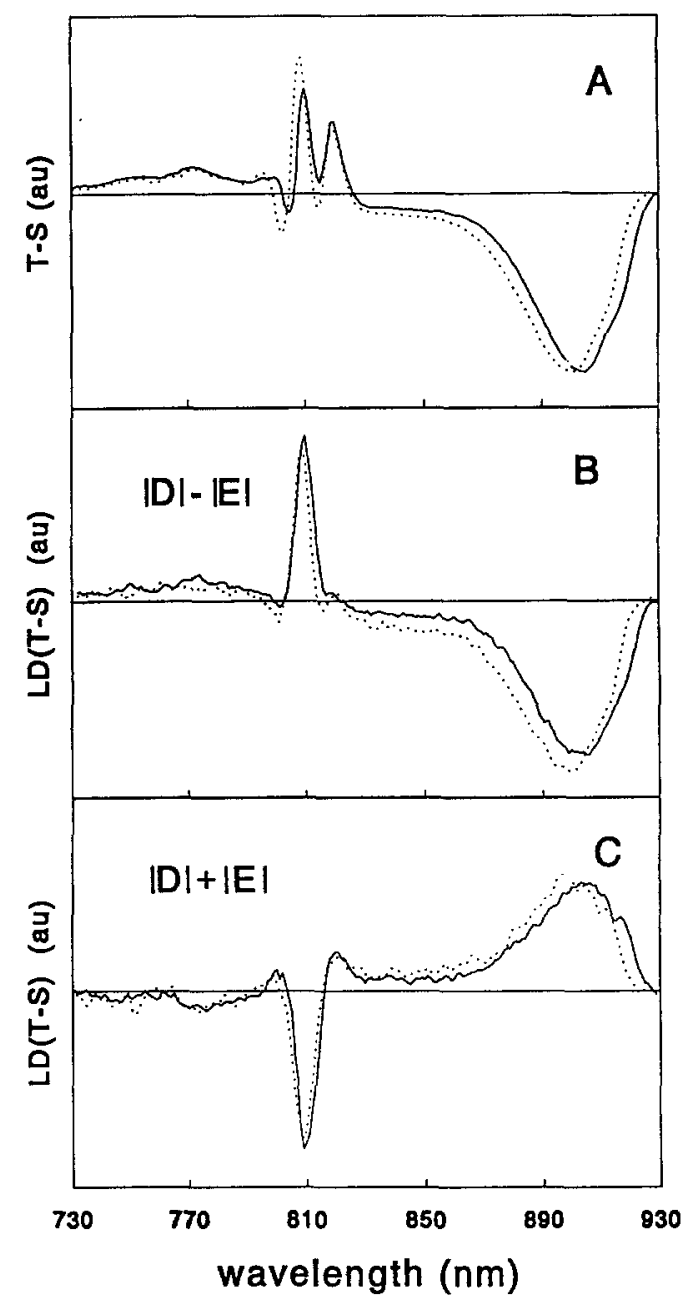

Fig. 5. (A) $(\mathrm{T}-\mathrm{S})$ spectra monitored at $463 \mathrm{MHz}$, (B) LD $(\mathrm{T}-\mathrm{S})$ spectra monitored at $463 \mathrm{MHz}$, (C) LD (T-S) spectra monitored at $656 \mathrm{MHz}$. for wild-type (dotted line) and mutant (solid line) RCs.

mutant has red-shifted by $4 \mathrm{~nm}$ compared to that of the wild type, both in the $T-S$ and in the absorption spectrum, measured under the same conditions.

The $L D(T-S)$ spectrum represents the difference between the $T-S$ spectrum measured with the polarization of the probe beam parallel and perpendicular to the magnetic field vector of the microwave field. The angle $\alpha_{i}$ between the optical transition moment $\mu^{\circ}$ of a given absorption band in the $\mathrm{T}-\mathrm{S}$ spectrum and the triplet spin axis $i$ can be determined from the ratio $\mathrm{R}_{i}$ between the amplitude of the $\mathrm{T}-\mathrm{S}$ and the $\mathrm{LD}(\mathrm{T}-\mathrm{S})$ signal at that wavelength, using (Hoff et al. 1985):

$$
\mathrm{R}_{\mathrm{i}}=\frac{\mathrm{LD}(\mathrm{T}-\mathrm{S})}{\mathrm{T}-\mathrm{S}}=\frac{3 \cos ^{2} \alpha_{\mathrm{i}}-1}{\cos ^{2} \alpha_{\mathrm{i}}+3} .
$$

The microwave power must be low enough to fulfill the condition of a truly random sample. Experimentally, this condition is met by measuring $\mathrm{R}_{i}$ as a function of microwave power and extrapolating to the zero-power limit. In RCs the experimentally accessible spin axes are the $y$ and $x$-axes, corresponding to the $|D|-|E|$ and the $|D|+|E|$ transition of $P^{T}$, respectively, where we take $D>0$ and $E>0$ as in Thurnauer and Norris (1977). Measurement of $R_{i}$ for two directions i (two zero-field transitions) fixes the optical transition moment in the $(x, y, z)$ microwave coordinate frame, to a fourfold degeneracy. Using the $|D|-|E|$ and the $|D|+|E|$ transitions of the mutant, we found for the $905 \mathrm{~nm}$ band $\mathrm{R}_{\mathrm{y}}=0.43$ and $\mathrm{R}_{\mathrm{x}}=0.28$, yielding $\alpha_{\mathrm{y}}=24^{\circ} \pm 2^{\circ}$ and $\alpha_{\mathrm{x}}=80^{\circ} \pm 5^{\circ}$. For the wild type the angles were: $\alpha_{y}=20^{\circ} \pm 2^{\circ}$ and $\alpha_{x}=85^{\circ} \pm 5^{\circ}$.

The shapes of the $\operatorname{LD}(\mathrm{T}-\mathrm{S})$ spectra of the mutant and the wild type in the 790-820 nm region are essentially the same. From this, and from the difference in shape of the two $T-S$ spectra, we can immediately conclude that the R-value(s), defined by Eq. (1), have changed for one or more absorption bands in the $790-820 \mathrm{~nm}$ region of the $T-S$ spectrum. Comparing the $\mathrm{T}-\mathrm{S}$ and the $\mathrm{LD}(\mathrm{T}-\mathrm{S})$ spectra, the change in the ratio $R_{x, y}$, measured at $808 \mathrm{~nm}$, could result from a change in the direction of the $808 \mathrm{~nm}$ optical transition moment, such that it is more parallel to the triplet $y$-axis and thus more perpendicular to the triplet $\mathrm{x}$-axis in the mutant compared to the wild type. Because positive and negative bands are strongly overlapping in the $790-820 \mathrm{~nm}$ region of the $\mathrm{T}-\mathrm{S}$ spectrum, however, this change in $\mathrm{LD}(\mathrm{T}-\mathrm{S}) / \mathrm{T}-\mathrm{S}$ value at $808 \mathrm{~nm}$ can also be explained by a smaller absolute $R$ value of the adjacent negative $806 \mathrm{~nm}$ and/or $814 \mathrm{~nm}$ bands.

\section{Discussion}

\section{Absorption spectra}

The absorption spectrum (Fig. 1) shows that several bands have shifted because of the muta- 
tion. This is best seen in the spectrum at $6 \mathrm{~K}$, where the bands are narrowed (Fig. 1B). Differences were found in the $Q_{y}$ and $Q_{x}$ regions of $\mathrm{BChl}$, as has been observed for other mutants (Gray et al. 1990, Mattioli et al. 1991). The $B C h l Q_{x}$ region of the absorption spectrum, with contributions of $B_{A}, B_{B}$ and $P$, shows a close similarity with the $800 \mathrm{~nm}$ region. On mutation, only the $802 \mathrm{~nm} \mathrm{BChl} \mathrm{band,} \mathrm{which} \mathrm{was} \mathrm{attribu-}$ ted to $\mathrm{B}_{\mathrm{A}}$ (Kirmaier et al. 1985), exhibited a red shift, whereas the energy levels of $B_{B}$ and $P$ remained unchanged. Assuming that the band shift in the $Q_{x}$ region correlates with that of the $Q_{y}$ region, we assign the red-shifting $597 \mathrm{~nm}$ band to $B_{A}$, and the unchanged $606 \mathrm{~nm}$ shoulder to $P$. A blue shift of $2 \mathrm{~nm}$ observed at the $547 \mathrm{~nm}$ band, attributed to $\Phi_{A}$, corresponded with the blue shift in the $Q_{y}$ region. The low-energy band of $\mathrm{P}$ at low temperature does not shift, suggesting that the mutation has no effect on the special pair. This is corroborated by the $|\mathrm{D}|$ and $|E|$ values of the triplet, obtained by ADMR measurements and by the value of the EPR linewidth of $P^{+}(9.7 \mathrm{G})$, which are the same for the wild type and the mutant.

\section{Fluorescence spectra and yields}

The low-temperature fluorescence spectra show efficient energy transfer from the chromophores to P (Fig. 2B). The strong fluorescence emission band at $926 \mathrm{~nm}$ could be due to a slower charge separation rate at $6 \mathrm{~K}$. Assuming that the steady state fluorescence emission is not due to inactive RCs (with longer lifetime of $\mathrm{P}^{*}$ ), one can draw conclusions on the temperature dependence of the electron transfer. The relative fluorescence yield of the mutant $\mathrm{RC}$ was four times lower at room temperature than at $6 \mathrm{~K}$, while the yield of the wild type $\mathrm{RC}$ was at $6 \mathrm{~K}$ at least 20 times lower than that of the mutant RC. For a temperature-independent fluorescence yield of the wild type (Woodbury and Parson 1984), the ratio of the fluorescence yield for mutant/wild type at room temperature is larger than 5. This estimate compares reasonably well with the strong increase in the lifetime of $\mathrm{P}^{*}$ going from the wild type to the mutant RCs. The four times higher fluorescence yield in the mutant at $6 \mathrm{~K}$ compared to room temperature, predicts that the lifetime of $\mathrm{P}^{*}$ increases to more than $100 \mathrm{ps}$ at $6 \mathrm{~K}$, making charge separation at least 50 times slower than that of the wild type at this temperature. This strong temperature dependence supports the idea that the electron transfer in the mutant is thermally activated at room temperature and that the reaction mechanism may change at low temperature, e.g. from a stepwise electron transfer to a superexchange mechanism.

\section{Structural changes}

Since tryptophan is a rather large residue, it could cause a structural change in the protein, which could influence the charge-separation rate in unpredictable ways. The measurements performed with ADMR spectroscopy give us some information on these structural changes, if any.

The ZFS-parameters and the decay rates of the sublevels of the primary donor triplet depend on the structure and the environment of $\mathrm{P}$. Both observables have virtually the same values for the mutant and the wild type, indicating that on mutation there are no changes in the structure and the environment of the primary donor that influences its properties in the triplet state. A small difference in the $\operatorname{LD}(\mathrm{T}-\mathrm{S}) /(\mathrm{T}-\mathrm{S})$ ratio for the mutant and the wild type might implicate that the angles between the optical transition moments contributing to the $790-820 \mathrm{~nm}$ region and the triplet axes have been altered slightly by the mutation (Fig. 4). The $802 \mathrm{~nm}$ band has been attributed mainly to $B_{A}$ with some contribution from $\Phi_{A}$ and the $814 \mathrm{~nm}$ band mainly to $B_{B}$, possibly with some contribution from the highenergy exciton component of $\mathbf{P}$ (Kirmaier et al. 1985). With this assignment, the shift of the $802 \mathrm{~nm}$ band (wild type) to longer wavelength ( $806 \mathrm{~nm}$ ) and the blue shift of the $\Phi_{\mathrm{A}}$ band can be attributed to a stronger exciton coupling between $B_{A}$ and $\Phi_{A}$. Upon triplet formation both the $806 \mathrm{~nm}$ band and the $814 \mathrm{~nm}$ shift to shorter wavelength, the $806 \mathrm{~nm}$ band to about $800 \mathrm{~nm}$ and the $814 \mathrm{~nm}$ band to $808 \mathrm{~nm}$. The $808 \mathrm{~nm}$ band appearing upon triplet formation is therefore attributed to $B_{B}$ and is not affected by the mutation. With this interpretation, it is unlikely that the apparent change in R-value observed at $808 \mathrm{~nm}$ band is caused by a change in R-value of the $808 \mathrm{~nm}$ band. It seems more 
probable that the adjacent negative $806 \mathrm{~nm}$ band gives rise to the observed changes in $\mathrm{R}$-value. The change in R-value of the $806 \mathrm{~nm}$ band should correspond to its transition moment becoming less parallel to the donor triplet y-axis. Since the optical transition moment of $P$ $(900 \mathrm{~nm})$ is almost parallel to this triplet y-axis, the optical transition moment of the $\mathrm{B}_{\mathrm{A}}$ (the main contributor to the $806 \mathrm{~nm}$ band) also becomes less parallel to the optical transition moment of $\mathrm{P}$ in the mutant compared to the wild type.

Summarizing, the absorption and the $\mathrm{T}-\mathrm{S}$ spectra could be simulated by an increase of the $\left(\mathrm{B}_{\mathrm{A}}, \Phi_{\mathrm{A}}\right)$ interaction. Furthermore, a less parallel alignment of the transition moment of $B_{A}$ and $P$ will decrease the interaction of $\left(P, B_{A}\right)$ without changing other parameters, such as zero-order energies and dipole strengths.

The change in the $\left(\mathrm{B}_{\mathrm{A}}, \Phi_{\mathrm{A}}\right)$ interaction can be explained by a difference in orientation or position of $B_{A}$ or $\Phi_{A}$. A displacement of $B_{A}$ towards $\Phi_{A}$ or vice versa will increase the $\left(B_{A}, \Phi_{A}\right)$ interaction. A rotation of the $Q_{Y}$ transition of the $\mathrm{B}_{\mathrm{A}}$ moment towards the $\mathrm{C}_{2}$-symmetry axis has the same effect. In the simple exciton model used, the influence of changes in the environment (amino acids) on the energies and the dipole strengths of the chromophores are not taken into account. Although such an indirect effect on the dipolar strength of $\mathrm{B}_{\mathrm{A}}$ or $\Phi_{\mathrm{A}}$ cannot be excluded, preliminary CP-MAS NMR results (Shochat et al., manuscript in preparation) indicate that no major changes occur in the ${ }^{13} \mathrm{C}$-labeled Tyr signals of the mutant.

Using molecular orbital calculations, Plato et al. (1988) explained the unidirectionality partially by an orbital overlap between $P$ and $B_{A}$ that is stronger than that between $P$ and $B_{B}$. In our description, the orbital overlap between $P$ and $B_{A}$ will be smaller for the mutant compared to the wild type, both for a displacement of $B_{A}$ towards $\Phi_{A}$, and a rotation of its $\mathrm{Q}_{\mathrm{Y}}$ transition moment towards the $\mathrm{C}_{2}$-symmetry axis. In the latter case, the optical $Q_{Y}$ transition moments of $\mathrm{P}$ and $\mathrm{B}_{\mathrm{A}}$ and, therefore, the macrocycle planes, are less parallel for the mutant than for the wild type. The resulting decrease of orbital overlap of $P$ and $B_{A}$ might lower the rate of charge separation in the $\mathrm{L}$-chain.

\section{Charge-separation kinetics}

The replacement of tyrosine (M)Y210 by a tryptophan affects the initial electron transfer step significantly. Room-temperature sub-picosecond spectroscopy shows that the decay of $\mathbf{P}^{*}$ is bi-exponential, with a main, slow time constant of $36 \mathrm{ps}$ and a weak, additional, fast time constant of 5.1 ps (Fig. 3). Similar time constants were observed at $660 \mathrm{~nm}$, which is the probing wavelength for the formation of the charge-separated $\mathrm{P}^{+} \Phi_{\mathrm{A}}^{-}$state (data not shown). Comparing the charge-separation rates with those of previously reported, analogous mutants (Gray et al. 1990, Nagarajan et al. 1990, Chan et al. 1991, Hamm et al. 1993), the (M)Y210W mutant appears to have the slowest charge-separation rate component of all reported mutants to date. A similar result (a time constant of $35 \pm 3$ ps) was reported by Nagarajan et al. (1992) for the same mutation. This component is more than ten times slower than in the wild type (assuming a single exponential decay). Nagarajan et al. (1992) found a qualitative correlation between the increase in the midpoint redox potential for the oxidation of $P$ and the slow-down of the electron transfer in several mutants including the (M)Y210W mutant. It is possible that the slow charge-separation rate results from two effects, a change in redox potential of $P$ (caused, for example, by the different polarizability and local charge density of the tryptophan, Parson et al. (1990)) and the decrease in orbital overlap of $P$ and $\mathrm{B}_{\mathrm{A}}$, suggested by the ADMR measurements. Alternatively, the replacement of tyrosine (M)Y210 by tryptophan could alter the energy level of the intermediate state $\mathrm{P}^{+} \mathrm{B}_{\mathrm{B}}^{-}$(see for example Parson et al. (1990)), and thereby slow down the charge-separation rate. This could also lead to the involvement of a parking state $\mathrm{P}^{+} \mathrm{B}_{\mathrm{B}}^{-}$.

Bi-exponential kinetics of the time-resolved absorption have been found at $920 \mathrm{~nm}$ for $R b$. sphaeroides and $R b$. capsulatus mutants in which tyrosine-(M) 210 had been exchanged by phenylalanine (Finkele et al. 1990, Chan et al. 1991, Hamm et al. 1993), and leucine (Finkele et al. 1990). But for most mutations on the tyrosine(M) 210 site, the kinetics at room temperature were fitted with single exponentials (Nagarajan et al. 1990, Chan et al. 1991). Recent studies on 
femtosecond spontaneous- and stimulated-emission studies have shown that for all RCs studied ( $R b$. sphaeroides R-26, (Du et al. 1992); (M)Y210P, (L)P181Y and the corresponding double mutant (Hamm et al. 1993), Chloroflexus aurantiacus (Martin et al. 1990, Feick et al. 1990, Becker et al. 1991, Müller et al. 1992, Hamm et al. 1992, Hamm et al. 1993) and $R b$. capsulatus wild type, (M)Y210P, (L)P181Y and the corresponding double mutant ( $\mathrm{Du}$ et al. 1992)), the kinetics of the decay of the spontaneous emission of $\mathrm{P}^{*}$ are bi-modal.

Nagarajan et al. (1990) explained the nonexponential reaction kinetics in terms of a conformational heterogeneity in the sample, induced by a lack of stabilizing influence of the rigid aromatic ring or the phenolic group upon mutation. They suggested that this effect might be more evident at low temperatures where interconversion among different conformational states would occur relatively slowly. Since tryptophan is a relatively bulky residue, it is hard to conceive two different conformations of the protein that could easily interconvert. Moreover, ${ }^{13} \mathrm{C}$ MAS-NMR spectra of RCs of the (M)Y210W mutant do not suggest the existence of two distinctly different conformations (S. Shochat et al., manuscript in preparation). Therefore, this kind of two-state heterogeneity does not seem to apply in our case. It is not excluded, however, that more subtle heterogeneities, leading to a distribution of many slightly different states, are the cause of the apparant biphasic kinetics. Kirmayer and Holten (1990) suggested a distribution of RCs with different rates of electron transfer. If the different RCs in the distribution interconvert on a time scale longer than that of the primary photochemistry, then this would lead to non-exponential kinetics of the primary electron transfer. As a possible cause for such a inhomogeneous distribution, differences in the degree of hydrogen bonding between the C-10 keto group of $\Phi_{\mathrm{A}}$ and the adjacent glutamic acid residue (Glu L104) were cited. Based on spontaneous emission studies, Norris and Fleming (personal communication) also suggested that a distribution of conformations could lead to an apparent biphasic decay pattern of the emission. Computer simulations (R. van der Vos, unpublished results) indicate that a Gaussian distribution of the difference in free energy $\Delta G$ between the initial and the product states, which is related to the charge separation by the Marcus equation (Marcus 1956), indeed may result in a decay pattern that can be fitted with a bi-exponential function, within the signal-to-noise ratio of the ps-measurements. Evidence for heterogeneity of $P$ conformation was also reported by Den Blanken et al. (1983), who observed in ADMR experiments a pronounced dependence of the primary donor bleaching in the triplet-singlet difference spectrum on the applied microwave field. A contrasting view was advanced by Lyle et al. (1993), who on the basis of hole-burning studies proposed that the role inhomogeneities involving the structure or energetics of $\mathrm{P}$, play in the charge-separation kinetic, is limited.

Finally, the biphasic decay of $\mathrm{P}^{*}$ could be explained by assuming that an additional intermediate, a so-called parking state, is involved. Time-resolved fluorescence experiments showing bi-exponential decay of the excited primary donor in wild type RCs of $R b$. sphaeroides, suggested to Müller et al. (1992) and Hamm et al. (1993) that such a state could involve chromophores on the inactive B-branch. Temporarily parking the excitation energy of the excited primary donor in the inactive B-branch by either energy transfer, or by formation of a real chargeseparated state $\mathrm{P}^{+} \mathrm{B}_{\mathrm{B}}^{-}$or $\mathrm{P}^{+} \Phi_{\mathrm{B}}^{-}$, results in biexponential decay of the excited primary donor.

\section{Conclusions}

We have shown that the charge-separation process in the RC of the (M)Y210W mutant of $R b$. sphaeroides showed bi-exponential kinetics at room temperature with a main time constant of $36 \mathrm{ps}$ and an additional fast time constant of $5.1 \mathrm{ps}$. The fluorescence yield of these RCs was four times higher at $6 \mathrm{~K}$ than at room temperature. This could be due to an increase in the lifetime of $\mathrm{P}^{*}$ at cryogenic temperatures. EPR and ADMR measurements indicate that the dimeric structure of $\mathbf{P}$ is not altered significantly by the mutation. In contrast, the interaction of the accessory bacteriochlorophyll $\mathrm{B}_{\mathrm{A}}$ with its environment appears to be altered in the mutant, 
possibly because of a change in its position. This could be one of the factors that causes the slower charge-separation rate in the mutant.

\section{Acknowledgments}

This work was supported by The Netherlands Foundations for Chemical Research (SON) and for Biophysics, financed by The Netherlands Organization for Scientific Research (NWO) and by Twinning Grant No SC 1-CT90-0569 of the European Community. P.G. is a research fellow of the Royal Netherlands Academy of Arts and Sciences. We are grateful to Jan Schutrups and Saskia Jansen for their help with RC preparations and to H.J.M. dé Groot for helpful discussions. S.S. thanks Prof L. Bosch and other members of the Department of Biochemistry, Leiden University, for their hospitality.

\section{References}

Allen JP, Feher G, Yeates TO, Komiya $\mathrm{H}$ and Rees DC (1987a) Structure of the reaction center from Rhodobacter sphaeroides R-26: The cofactors. Proc Natl Acad Sci USA 84: $5730-5734$

Allen JP, Feher G, Yeates TO, Komiya $H$ and Rees DC (1987b) Structure of the reaction center from Rhodobacter sphaeroides R-26: The protein subunits. Proc Natl Acad Sci USA 84: 6162-6166

Becker M, Nagarajan V, Middendorf D, Parson WW, Martin JE and Blankenship RE (1991) Temperature dependence of the initial electron-transfer kinetics in photosynthetic reaction centers of Chloroflexus aurantiacus. Biochim Biophys Acta 1057: 299-312

Breton J, Martin JL, Migus A, Antonetti A and Orszag A (1986) Femtosecond spectroscopy of excitation energy transfer and initial charge separation in the reaction center of the photosynthetic bacterium Rhodopseudomonas viridis. Proc Natl Acad Sci USA 83: 5121-5125

Chan CK, Chen LXQ, Dimagno TJ, Hanson DK, Nance SL, Schiffer M, Norris JR and Fleming GR (1991) Initial electron transfer in photosynthetic reaction centers of Rhodobacter capsulatus mutants. Chem Phys Lett 176: 366-372

Den Blanken HJ and Hoff AJ (1983) Resolution enhancement of the triplet-singlet absorbance difference spectrum and the triplet-ESR spectrum in zero field by the selection of sites. An application to photosynthetic reaction centers. Chem Phys Lett 98: 255-261

Den Blanken HJ, Meiburg RF and Hoff AJ (1984) Polarized triplet-minus-singlet absorbance difference spectra measured by absorbance-detected magnetic resonance. An application to photosynthetic reaction centres. Chem Phys Lett 105: 336-342

Dressler K, Umlauf E, Schmidt S, Hamm P, Zinth W, Buchanan S and Michel H (1991) Detailed studies of the subpicosecond kinetics in the primary electron transfer of reaction centers of Rhodopseudomonas viridis. Chem Phys Lett 183: $270-276$

Du M, Rosenthal SJ, Xie X, DiMagno TJ, Schmidt M, Hanson DK, Schiffer M, Norris JR and Fleming GR (1992) Femtosecond spontaneous-emission studies of reaction centers from photosynthetic bacteria. Proc Natl Acad Sci USA 89: 8517-8521

Feick R, Martin JL, Breton J, Volk M, Scheidel G, Langenbacher T, Urbano C, Ogrognik A and Michel-Beyerle ME (1990) Biexponential charge separation and monoexponential decay of $\mathrm{P}^{+} \mathrm{H}^{-}$in reaction centers of Chloroflexus aurantiacus. In: Michel-Byerle ME (ed) Reaction Centers of Photosynthetic Bacteria, pp 181-188, Springer Series in Biophysics Vol 6. Springer-Verlag, Heidelberg

Finkele U, Lauterwasser C, Zinth W, Gray KA and Oesterhelt D (1990) Role of tyrosine M210 in the initial charge separation of reaction centers of Rhodobacter sphaeroides. Biochemistry 29: 8517-8521

Fischer MR, De Groot HJM, Raap J, Winkel C, Hoff AJ and Lugtenburg J (1992) ${ }^{13} \mathrm{C}$ magic angle spinning NMR study of the light-induced and temperature-dependent changes in Rhodobacter spaeroides R26 reaction centers enriched in $\left[4^{\prime}-{ }^{13} \mathrm{C}\right]$ tyrosine. Biochemistry 31: $11038-11049$

Gast $P$ and Hoff AJ (1979) Transfer of light-induced electron-spin polarization from the intermediate acceptor to the prereduced primary acceptor in the reaction center of photosynthetic bacteria. Biochim Biophys Acta 548: 520535

Gray KA, Farchaus JW, Wachtveitl J, Breton J and Oesterhelt D (1990) Initial characterization of site-directed mutants of tyrosine $\mathrm{M} 210$ in the reaction center of Rhodobacter sphaeroides. EMBO J 9: 2061-2070

Hamm P, Gray KA, Oesterhelt D, Feick R, Scheer H and Zinth W (1993) Subpicosecond emission studies of bacterial reaction centers. Biochim Biophys Acta 1142: 99-105

Hoff AJ (1982) ODMR Spectroscopy in Photosynthesis II. The Reaction Center Triplet in Bacterial Photosynthesis. In: Clark RH (ed) Triplet State ODMR Spectroscopy, pp 367-425. John Wiley \& Sons, New York

Hoff AJ, den Blanken HJ, Vasmel H and Meiburg RF (1985) Linear-dichroic triplet-minus-singlet absorbance difference spectra of reaction centers of the photosynthetic bacteria Chromatium vinosum, Rhodopseudomonas sphaeroides $\mathbf{R}$ 26 and Rhodospirillum rubrum. Biochim Biophys Acta 806: 389-397

Holzapfel W, Finkele U, Kaiser W, Oesterhelt D, Scheer H, Stilz HU and Zinth W (1990) Initial electron-transfer in the reaction center from Rhodobacter sphaeroides. Proc Natl Acad Sci USA 87: 5168-5172

Kirmaier C and Holten D (1987) Primary photochemistry of reaction centers from the photosynthetic purple bacteria. Photosynth Res 13: 225-260

Kirmaier C, Holten D and Parson WW (1985) Picosecondphotodichroism studies of the transient states in Rhodopseudomonas sphaeroides reaction centers at $5 \mathrm{~K}$. Effects 
of electron transfer on the six bacteriochlorin pigments. Biochim Biophys Acta 810: 49-61

Kunkel TA (1987) Rapid and efficient site-specific mutagenesis without phenotypic selection. Proc Natl Acad Sci USA 82: 488-492

Lyle PA, Kolaczkowski SV and Small GJ (1993) Photochemical hole-burning spectra of protonated and deuterated reaction centers of Rhodobacter sphaeroides. J Phys Chem 97: 6924-6933

Marcus RA (1956) On the theory of oxidation-reduction reactions involving electron transfer. I. J Chem Phys 24: 966-978

Martin JL, Lambry JC, Ashokkumar M, Michel-Beyerle ME, Feick R and Breton J (1990) Primary charge separation in reaction centers of Chloroflexus aurantiacus bacterium. In: Harris CB, Ippen EP, Mourruo GA and Zewail AH (eds) Ultrafast Phemonema VII, pp 524-528. Springer Series in Chemical Physics, Vol 53. SpringerVerlag, Heidelberg

Mattioli TA, Gray KA, Lutz M, Oesterhelt D and Robert B (1991) Resonance Raman Characterization of Rhodobacter sphaeroides reaction centers bearing site-directed mutations at tyrosine M210. Biochemistry 30: 1715-1722

Michel H, Epp O and Deisenhofer J (1986) Pigment-protein interactions in the photosynthetic reaction centre from Rhodopseudomonas viridis. EMBO J 5: 2445-2451

Müller MG, Griebenow K and Holzwarth AR (1992) Primary processes in isolated bacterial reaction centers from Rhodobacter-sphaeroides studied by picosecond fluorescence kinetics. Chem Phys Lett 199: 465-469

Nagarajan V, Parson WW, Gaul D and Schenck C (1990) Effect of specific mutations of tyrosine-(M)210 on the primary photosynthetic electron-transfer process in Rhodobacter sphaeroides. Proc Natl Acad Sci USA 87: 7888-7892

Nagarajan V, Davis D, Parson W and Schenck C (1992) Abstracts of the Cadarache NATO Workshop on 'Structure, Function and Dynamics of the Bacterial Reaction Center', May 10-15

Otte SCM, Vanderheiden JC, Pfennig N and Amesz J (1991) A comparative study of the optical characteristics of intact cells of photosynthetic green sulfur bacteria containing bacteriochlorophyll $c, d$ or $e$. Photosynth Res 28: 77-87

Paddock ML, Rongey SH, Feher G and Okamura MY (1989) Pathway of proton transfer in bacterial reaction centers: Replacement of glutamic acid 212 in the $\mathrm{L}$ subunit by glutamine inhibits quinone (secondary acceptor) turnover. Proc Natl Acad Sci USA 86: 6602-6606

Parson WW, Chu Z-T and Warshel A (1990) Electrostatic control of charge separation in bacterial photosynthesis. Biochim Biophys Acta 1017: 251-272

Plato $M$, Mobius $K$, Michel-Beyerle ME, Bixon $M$ and Jortner J (1988) Intermolecular electronic interactions in the primary charge separation in bacterial photosynthesis. J Am Chem Soc 110: 7279-7285

Schelvis JPM, Liu BL, Aartsma TJ and Hoff AJ (1992) The electron transfer rate from $B \mathrm{BP}_{\mathrm{A}}$ to $\mathrm{Q}_{\mathrm{A}}$ in reaction centers of Rhodobacter sphaeroides R-26 - Influence of the $\mathrm{H}$ subunit, the $\mathrm{Q}_{\mathrm{A}}$ and $\mathrm{Fe}-2$ + cofactors, and the isoprene tail of $\mathrm{Q}_{\mathrm{A}}$. Biochim Biophys Acta 1102: 229-236

Scherer POJ and Fischer SF (1987) Model studies to lowtemperature optical transitions of photosynthetic reaction centers. II. Rhodobacter sphaeroides and Chloroflexus aurantiacus. Biochim Biophys Acta 891: 157-164

Schmidt S, Arlt T, Hamm P, Lauterwasser C, Finkele U, Drews $G$ and Zinth W (1993) Time-resolved spectroscopy of the primary photosynthetic processes of membranebound reaction centers from an antenna-deficient mutant of Rhodobacter capsulatus. Biochim Biophys Acta 1144: 385-390

Shiozawa JA, Lottspeich F, Oesterhelt D and Feick R (1989) The primary structure of the Chloroflexus aurantiacus reaction-center polypeptides. Eur J Biochem 180: 75-84

Shochat S, Van Noort PI, Van der Vos R, Otte SCM, Schelvis $H$, Vrieze J, Kleinherenbrink FAM, Gast $P$ and Hoff $A J$ (1992) Spectrosocopic characterization of reaction centers of the $\mathrm{M}^{\text {tyr } 210 \rightarrow \operatorname{tr}}$ mutant of Rhodobacter sphaeroides. In: Murata $N$ (ed) Proceedings of the IXth International Congress on Photosynthesis, pp 413-416. Kluwer Academic Publishers, Dordrecht

Thurnauer MC and Norris JR (1977) The ordering of the zero field triplet spin sublevels in the chlorophylls. A magnetophotoselection study. Chem Phys Lett 47: 100-112

Visser JWM (1975) Photosynthetic reactions at low temperatures. PhD thesis, Leiden University

Wang RT and Clayton RK (1971) The absolute yield of bacteriochlorophyll fluorescence in vivo. Photochem Photobiol 13: 215-224

Williams JC, Steiner LA and Feher G (1986) Primary structure of the reaction center from Rhodopseudomonas sphaeroides. Proteins: Structure, Function and Genetics 1: 312-325

Woodbury NWT and Parson WW (1984) Nanosecond fluorescence from isolated photosynthetic reaction centers of Rhodopseudomonas sphaeroides. Biochim Biophys Acta 767: 315-361 